\title{
Robust and Adaptive Feedback Noise Attenuation in Ducts
}

\author{
Ioan Doré Landau, Raúl Meléndez, Luc Dugard, and Gabriel Buche
}

\begin{abstract}
In this paper the attenuation of sound propagation in an air-handling duct using robust and adaptive feedback active noise control strategies is investigated. The case of multiple narrow band disturbances located in distinct frequency regions and the interference occurring in the presence of disturbances with very close frequencies are considered. The active control uses a loudspeaker as compensatory system. The objective is to minimize the residual noise at the end of the duct segment considered. The system does not use any additional sensor for getting in real time information upon the disturbances. A hierarchical feedback approach will be used for the control of the system. At the first level a robust linear controller will be designed taking advantage of the knowledge of the domains of variation of the frequencies of the noise disturbances. To further improve the performance, a direct adaptive control algorithm will be added. Its design is based on the use of the internal model principle combined with the Youla-Kučera parametrization of the controller. Both robust and adaptive control require the knowledge of the discrete-time model of the secondary path (the transfer function between the control loudspeaker and the residual noise measurement) which is obtained by identification from experimental data. Experimental results on a relevant duct active noise control test bench will illustrate the performance of the proposed methodology.
\end{abstract}

Index Terms-Active noise control, System Identification, Internal model principle, Youla-Kučera parametrization, Adaptive control, Robust control.

\section{INTRODUCTION}

In most cases, feed-forward noise compensation is currently used for ANC when a disturbance's image is available (a correlated measurement with the disturbance). See [1], [2], [3], [4]. However, these solutions, inspired by Widrow's technique for adaptive noise cancellation, see [5], ignore the possibilities offered by feedback control systems and have a number of disadvantages: 1) they require the use of an additional transducer, 2) difficult choice for its location and, 3 ) in most cases, presence of a "positive" coupling between the compensatory system and the disturbance image's measurement, which can cause instabilities [4]. To achieve the attenuation of the disturbance without measuring it, a feedback solution can be considered. This is particularly suitable for attenuating multiple time-varying narrow band noise.

Residual noise can be described as the result of acoustic waves which pass trough the system, and the noise canceller's objective is to minimize it. In many cases, these waves can be

Financial support thanks to Consejo Nacional de Ciencia y tecnología de México, CONACYT.

The authors are with Univ. Grenoble Alpes, CNRS, Grenoble INP, GIPSA-lab, F-38000 Grenoble, France, (e-mail: Raul.Melendez, IoanDore.Landau, Luc.Dugard, Gabriel.Buche@gipsa-lab.grenoble-inp.fr). characterized in the frequency domain either as tonal disturbances or as narrow band disturbances, both with unknown and time-varying frequencies. The common framework is the assumption that a narrow band disturbance is the result of a white noise or a Dirac impulse passed through the "disturbance's model." More specifically, in discrete-time, the model for a single narrow-band or tonal disturbance is a notch filter with poles on the unit circle and zeros inside the unit circle (for details see [6]). In the context of this paper, robustness should be understood as performance robustness with respect to the variations of the characteristics of the disturbance noise. This will be achieved by using either a linear robust controller or an adaptive controller.

In managing the noise attenuation by feedback, the shape of the modulus of the output sensitivity function (the transfer function between the disturbance and the residual noise) is fundamental both from performance and robustness considerations. The output sensitivity function should be appropriately shaped, in order to avoid unwanted amplifications in the neighborhood of the frequencies of the disturbances which will be attenuated.

The problem of robust feedback noise attenuation in ducts by shaping the output sensitivity function has been addressed in [7]. The paper [8] considers the use of $H_{\infty}$ combined with LMI for a robust control design of noise attenuation in ducts. The paper [9] considers an $H_{\infty}$ approach to noise attenuation in headphones. If the frequency of the tonal or narrow band disturbances is known, the "internal model principle" can be used to achieve a very strong attenuation. However, since the frequencies of these noise disturbances vary, an adaptive approach has to be considered. The combination of the "internal model principle" (IMP) with the Youla-Kučera (YK) parametrization has allowed the development of a direct adaptive regulation scheme for active vibration control [6] and this approach will be used in this paper for active noise attenuation in ducts. This approach is different from the approaches considered in [10], [11], [12] which ignore IMP and YK parametrization and require a very large number of parameters to be adapted. One should mention the pioneering work of [13] in using IMP and YK parametrization. However the present paper uses different adaptation algorithms, a different design for the central controller and goes beyond the case of a single tonal disturbance.

Several problems have been considered in the field of active noise control. In this paper, one considers multiple unknown and time- varying tonal disturbances located within two distinct relatively small frequency ranges. To be specific, two cases will be considered: (i) the case of two time-varying 
tonal disturbances located in two distinct frequency regions and (ii) the case of four simultaneous tonal disturbances, two located in one limited frequency range and the other two in another frequency range. In this context, a very important problem is to be able to counteract the very low frequency oscillations (interference) which are generated when the two frequencies are very close. Since these disturbances are located within two relatively small frequency ranges, it is possible to consider a robust linear control design. The first case, in the context of active noise control in ducts was considered in [7] and the shaping of the output sensitivity function was achieved using the convex optimization procedure introduced in [14]. It will be shown in this paper that an elementary procedure for shaping appropriately the modulus of the sensitivity functions can be implemented by using stop band filters as shaping tools. For a basic reference on this approach, see [6].

To further improve the performance, an algorithm for direct adaptive rejection of the disturbances will be added [6]. This algorithm takes into account the IMP and uses the Youla-Kučera (YK) parametrization of the controller.

The real time performance of the noise cancellers depends upon the quality of the secondary path dynamic model used for designing the feedback control law. Despite long years of effort(See [15]. [16]), physical modeling is not relevant for obtaining good models for control design. What is needed in practice is a finite dimension discrete-time model which reproduces the system's dynamical behaviour. Once such a model is available, one can use digital control design techniques readily implementable on a real time computer. These models can be obtained directly from data using system identification techniques, see [17], [6], [7].

The paper is organized as follows. Section II describes the experimental setup. Section III presents briefly the equations describing the system model and the controller. Section IV summarizes the identification procedure and provides the model of the secondary path used in the controller design. Section $\mathrm{V}$ gives the specifications and the design of the robust controller. Section VI provides the algorithm used for adaptive disturbance rejection using the internal model principle. Section VII presents the experimental results obtained. Conclusions are given in Section VIII.

\section{EXPERIMENTAL SETUP}

The view of the test bench used for experiments is shown in Fig. 1 and its detailed scheme is given in Fig. 2.

The speaker used as the source of disturbances is labeled as 1, the control speaker is 2 and finally, at pipe's open end, the microphone that measures the system's output (residual noise) is denoted as 3 . The transfer function between the disturbance's speaker and the microphone $(1 \rightarrow 3)$ is denominated Primary Path, while the transfer function between the control speaker and the microphone $(2 \rightarrow 3)$ is denominated Secondary Path. Both speakers are connected to a xPC Target computer with Simulink Real Time ${ }^{\circledR}$ environment through a pair of high definition power amplifiers and a data acquisition card. $y(t)$ is the system's output (residual noise measurement), $u(t)$ is the control signal and $p(t)$ is the disturbance. Both primary and secondary paths have a double differentiator behaviour, since as input we have the voice coil displacement, and as output the air acoustic pressure. A second computer is used for development, design and operation with Matlab ${ }^{\circledR}$.

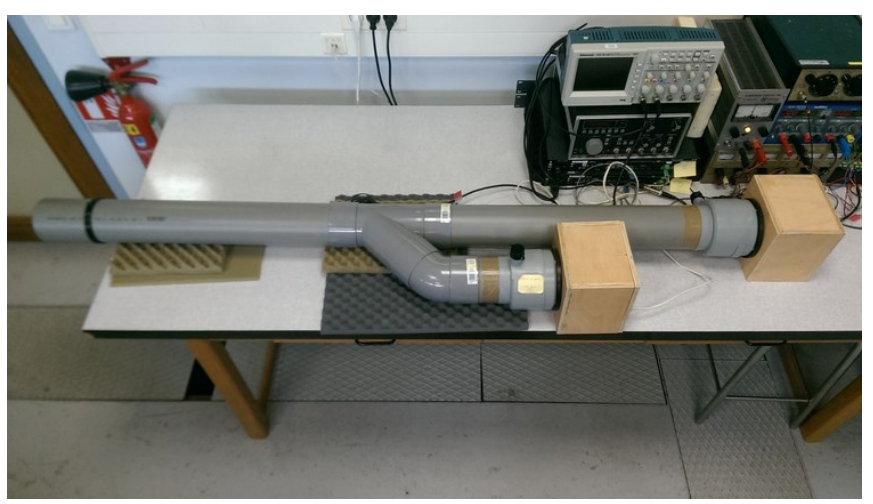

Fig. 1. Duct active noise control test bench (Photo).

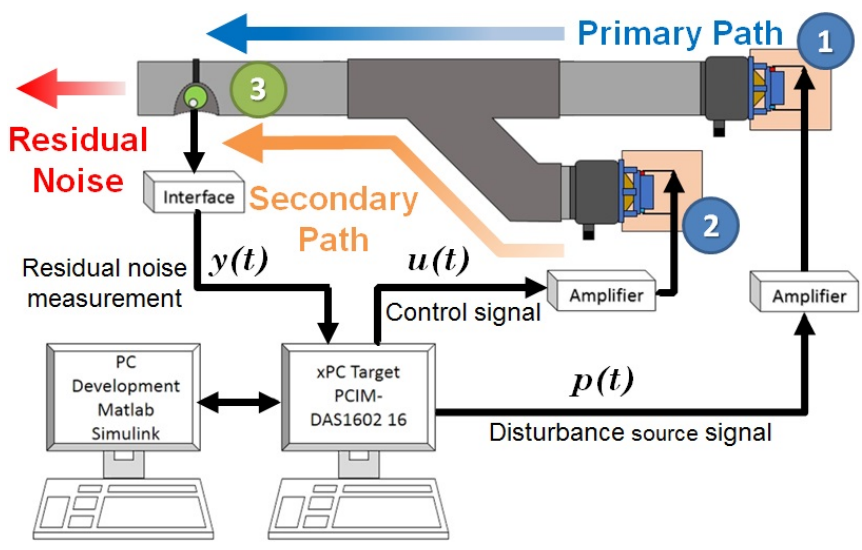

Fig. 2. Duct active noise control test bench diagram.

\section{SYSTEM DESCRIPTION}

The linear time invariant (LTI) discrete-time model of the secondary path, or plant, used for controller design is

$$
G\left(z^{-1}\right)=\frac{z^{-d} B\left(z^{-1}\right)}{A\left(z^{-1}\right)}=\frac{z^{-d} B^{\prime}\left(z^{-1}\right) D_{F}\left(z^{-1}\right)}{A\left(z^{-1}\right)},
$$

where $D_{F}\left(z^{-1}\right)=\left(1-z^{-1}\right)^{2}$ is a double differentiator filter and

$$
\begin{aligned}
A\left(z^{-1}\right) & =1+a_{1} z^{-1}+\cdots+a_{n_{A}} z^{-n_{A}}, \\
B^{\prime}\left(z^{-1}\right) & =b_{1} z^{-1}+\cdots+b_{n_{B^{\prime}}} z^{-n_{B^{\prime}},}
\end{aligned}
$$

with $d$ as the plant pure time delay in number of sampling periods ${ }^{1}$. The system's order (without the double differentiator) is:

$$
n=\max \left(n_{A}, n_{B^{\prime}}+d\right)
$$

\footnotetext{
${ }^{1}$ The complex variable $z^{-1}$ is used to characterize the system's behavior in the frequency domain and the delay operator $q^{-1}$ for the time domain analysis.
} 


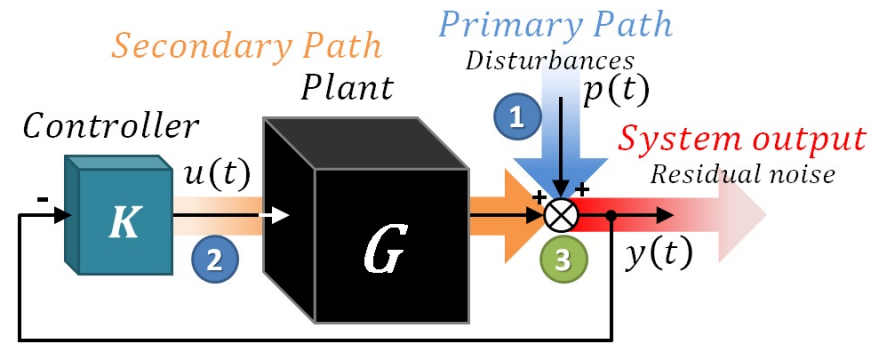

Fig. 3. Feedback regulation scheme.

Fig. 3 shows the closed loop feedback regulation scheme, where the controller $K$ is described by

$$
K\left(z^{-1}\right)=\frac{R}{S}=\frac{r_{0}+r_{1} z^{-1}+\cdots+r_{n_{R}} z^{-n_{R}}}{1+s_{1} z^{-1}+\cdots+s_{n_{S}} z^{-n_{S}}} .
$$

The plant's output $y(t)$ and the input $u(t)$ may be written as (see Fig. 3):

$$
\begin{aligned}
y(t) & =\frac{q^{-d} B\left(q^{-1}\right)}{A\left(q^{-1}\right)} \cdot u(t)+p(t), \\
S\left(q^{-1}\right) \cdot u(t) & =-R\left(q^{-1}\right) \cdot y(t) .
\end{aligned}
$$

In Eq. (6), $p(t)$ is the disturbances' effect on the measured output ${ }^{2}$ and $R\left(z^{-1}\right), S\left(z^{-1}\right)$ are polynomials in $z^{-1}$ having the following expressions:

$$
\begin{gathered}
R=H_{R} \cdot R^{\prime}=H_{R} \cdot\left(r_{0}^{\prime}+r_{1}^{\prime} z^{-1}+\ldots+r_{n_{R^{\prime}}}^{\prime} z^{-n_{R^{\prime}}}\right), \\
S=H_{S} \cdot S^{\prime}=H_{S} \cdot\left(1+s_{1}^{\prime} z^{-1}+\ldots+s_{n_{S^{\prime}}}^{\prime} z^{-n_{S^{\prime}}}\right),
\end{gathered}
$$

where $H_{S}\left(z^{-1}\right)$ and $H_{R}\left(z^{-1}\right)$ represent prespecified parts of the controller (used for example to incorporate the internal model of a disturbance, or to open the loop at some frequencies) and $S^{\prime}\left(z^{-1}\right)$ and $R^{\prime}\left(z^{-1}\right)$ are, in the present context, solutions of the Bezout equation:

$$
P=\left(A \cdot H_{S}\right) \cdot S^{\prime}+\left(z^{-d} B \cdot H_{R}\right) \cdot R^{\prime}
$$

In Eq. (10), $P\left(z^{-1}\right)$ represents the characteristic polynomial, which specifies the desired closed loop poles of the system.

The transfer functions between the disturbance $p(t)$ and the system's output $y(t)$ and the control input $u(t)$, denoted respectively output sensitivity and input sensitivity functions, are given by

$$
S_{y p}\left(z^{-1}\right)=\frac{A\left(z^{-1}\right) S\left(z^{-1}\right)}{P\left(z^{-1}\right)}
$$

and

$$
S_{u p}\left(z^{-1}\right)=-\frac{A\left(z^{-1}\right) R\left(z^{-1}\right)}{P\left(z^{-1}\right)},
$$

\section{System IDEnTIFication}

The design of the active noise control requires the knowledge of the dynamic model of the compensator system (the secondary path). This model will be obtained by system identification from experimental data (see [6], [17]).

\footnotetext{
${ }^{2}$ The disturbance passes through the primary path, and $p(t)$ is its output.
}

For design and application reasons (the objective is to reject tonal disturbances up to $400 \mathrm{~Hz}$ ), the sampling frequency was selected as $f_{s}=2500 \mathrm{~Hz}\left(T_{s}=0.0004 \mathrm{~s}\right)$ i.e. approximatively 6 times the maximum frequency to attenuate, in accordance with recommendation given in [6].

The characteristics of the Pseudo Random Binary Sequences (PRBS) used as excitation signal are: magnitude $=$ $0.15 \mathrm{~V}$, register length $=17$, frequency divider of 1 , sequence length: $2^{17}-1=131,071$ samples, guaranteeing a uniform power spectrum from about $70 \mathrm{~Hz}$ to $1250 \mathrm{~Hz}$. Since the transfer functions have a double differentiator behavior (input: speaker's coil position, output: acoustic pressure), this is considered as a system's known part and the objective is to identify the unknown part only. To do this, the input sequence is filtered by a double discrete-time differentiator $D_{F}=\left(1-q^{-1}\right)^{2}$ such that $u^{\prime}(t)=D_{F} \cdot u(t)$. The double differentiator will be concatenated with the identified model of the unknown part in the final models.

The next step in the identification procedure is the estimation of the order $\mathrm{n}$ of the model from the experimental data. The method of Duong ([6], [18]) has been used. Once an estimated order $\hat{n}$ is selected, one can apply a similar procedure to estimate $\hat{n}_{A}, \hat{n}-\hat{d}$, and $\hat{n}_{B^{\prime}}+\hat{d}$, from which $\hat{n}_{A}, \hat{n}_{B^{\prime}}$ and $\hat{d}$ are obtained. The estimated order $\mathrm{n}$ is selected as the value which minimizes a certain criterion. The value of $\hat{n}=36$ has been obtained but since the minimum was relatively flat, nearby values have also been considered. The final selection has been done by checking what order allows 1) to capture all the oscillatory modes in the model 2) to lead to the best statistical validation once the parameters are identified. Finally a model with an estimated order $n=40$ which led to $n_{A}=38, n_{B}^{\prime}=30$ and $d=8$ has been chosen.

Comparative parameter estimation considering various plant+noise models and estimation algorithms led to the conclusion that an ARMAX model representation is the most appropriate for this system and the best results in terms of statistical validation (whiteness test on he residual eror) have been obtained using the Output error with extended prediction model (termed OEEPM or XOLOE). See [6] for the detail of the methodology. Therefore the OEEPM model $n_{A}=38, n_{B}^{\prime}=30$ and $d=8$ has been chosen. It has 18 oscillatory modes with damping comprised between 0.0097 and 0.3129 . It has also 13 pairs of stable and unstable oscillatory zeros with damping comprised between -0.0159 and 0.5438 . The very low damped complex zeros and the unstable zeros are located in the frequency domain over $500 \mathrm{~Hz}$. The presence of these low damped zeros make the control system's design difficult. Fig. 4 gives the frequency characteristics of the identified complete models for the primary and secondary paths ${ }^{3}$.

\section{Robust Control Design}

\section{Control specifications}

The controller is designed to attenuate frequencies around $170 \mathrm{~Hz}$ and $285 \mathrm{~Hz}$, with a $\pm 5 \mathrm{~Hz}$ tolerance. Attenuation must

\footnotetext{
${ }^{3}$ Primary path model has been identified using the same procedure. This model is used for simulations only
} 


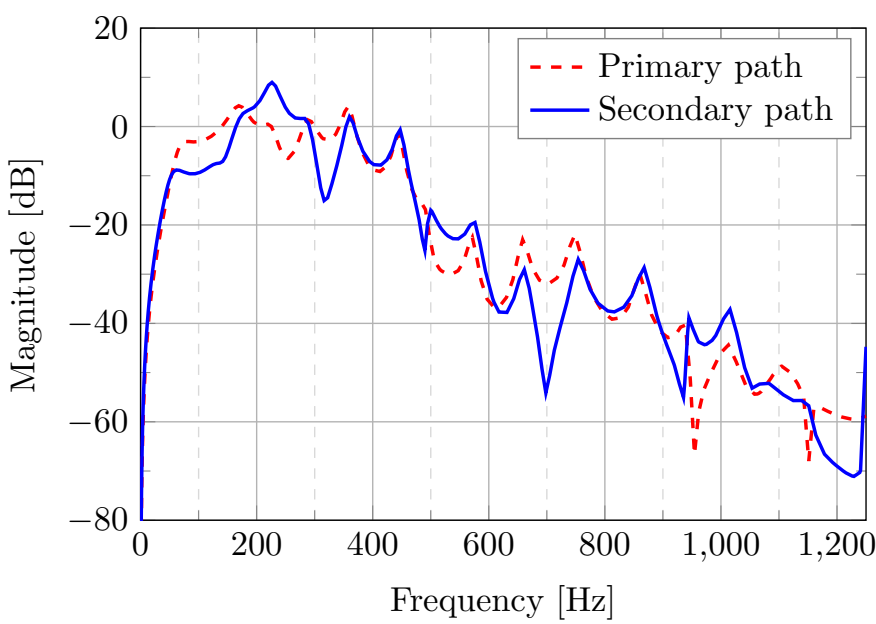

Fig. 4. Frequency characteristics of the identified primary and secondary paths models

be at least of $-18 \mathrm{~dB}$ in these regions and any undesired amplification should be less that $6 \mathrm{~dB}$. Also since the gain of the model is low over $600 \mathrm{~Hz}$, and very low damped complex zeros are present in high frequencies, the magnitude of the input sensitivity function should be below $-20 \mathrm{~dB}$ at frequencies over $600 \mathrm{~Hz}$ (in order to improve robustness with respect to additive uncertainties and avoid unnecessary control effort)

In addition, the controller's gain should be zero at $0 \mathrm{~Hz}$ since the plant does not have gain at zero frequency and the controller's gain should be zero also at the Nyquist frequency $\left(0.5 f_{s}\right)$, for robustness reasons (the unstable zeros are close to $0.5 f_{S}$ ). These control specifications will be achieved through the sensitivity functions' shaping.

\section{Design procedure}

To achieve the constraints at $0 \mathrm{~Hz}$ and at $0.5 f_{s}$ a fixed part $\left(H_{R}\right)^{4}$ is introduced in the controller:

$$
H_{R}\left(q^{-1}\right)=\left(1-q^{-1}\right)\left(1+q^{-1}\right)=1-q^{-2},
$$

The use of auxiliary poles is done such that the characteristic polynomial takes the form

$$
P\left(z^{-1}\right)=P_{D}\left(z^{-1}\right) \cdot P_{F}\left(z^{-1}\right),
$$

where $P_{D}$ are the dominant poles corresponding to the poles of the identified dynamic model, and $P_{F}$ are the auxiliary poles determined by the design requirements.

It is shown in [6] that a very accurate shaping of the output or the input sensitivity functions can be obtained by the use of 2nd order band-stop filters (BSF) of the form: $\frac{H_{S_{i}}\left(z^{-1}\right)}{P_{F S_{i}}\left(z^{-1}\right)}$ and respectively $\frac{H_{R_{i}}\left(z^{-1}\right)}{P_{F R_{i}}\left(z^{-1}\right)}$. Depending on whether the filter is designed for shaping the output or the input sensitivity function, the numerator of the discretized filter is included in the fixed part of the controller denominator $H_{S_{0}}$ or numerator $H_{R_{0}}$, respectively. The filter denominator is always included

\footnotetext{
${ }^{4} H_{R_{i}}, H_{S_{i}}, P_{F R_{i}}, P_{F S_{i}}$ will denote any given controller's fixed part.
}

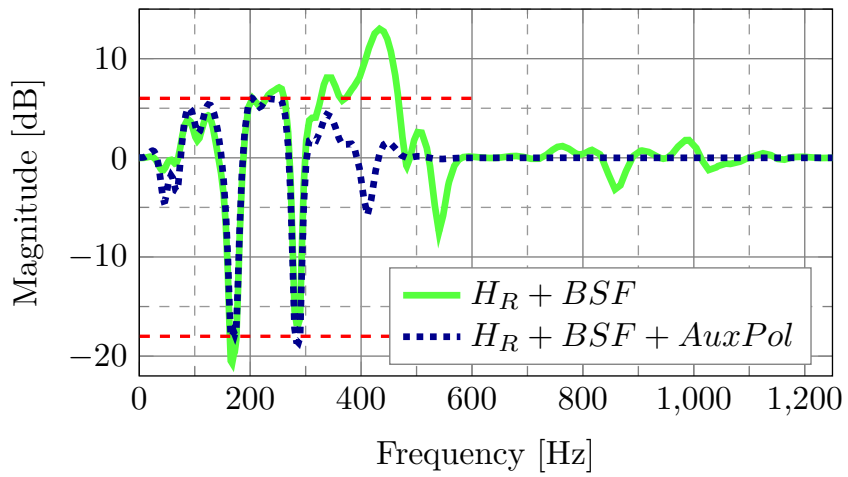

Fig. 5. Robust controller design, Output sensitivity function evolution.

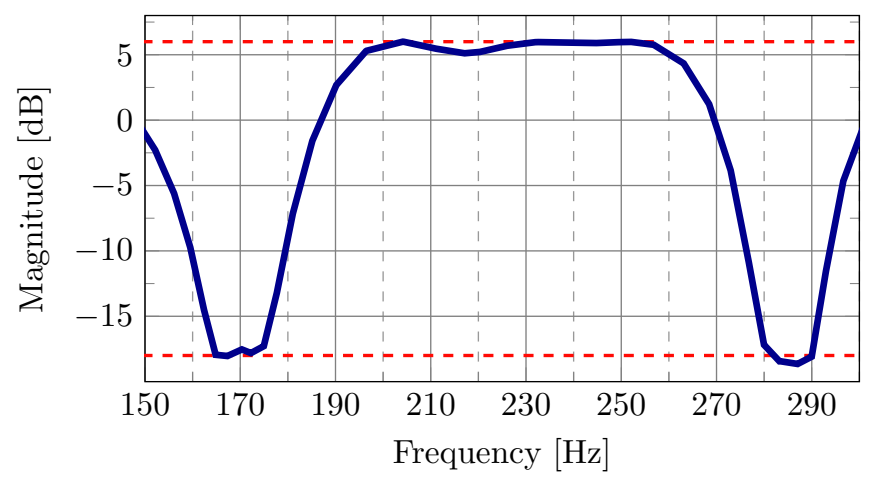

Fig. 6. Robust controller's output sensitivity function evolution, zoom.

in the closed loop characteristic polynomial. As such, the filter denominator influences the design of the controller indirectly in the computation of $S^{\prime}$ and $R^{\prime}$ as solutions of the Bezout equation (10).

The steps for the linear controller's design are:

1) include all (stable) secondary path poles in the closed loop characteristic polynomial.

2) open the loop at $0 \mathrm{~Hz}$ and at $1250 \mathrm{~Hz}$ by setting the fixed part of the controller numerator as in Eq. (13).

3) $9 \mathrm{BSF}$ on $S_{y p}$ have been used around each of the frequencies where attenuation is desired in order to assure the desired attenuation within $\pm 5 \mathrm{~Hz}$.

4) 8 BSF have been used on $S_{u p}$ to reduce its magnitude above $600 \mathrm{~Hz}$

5) to improve robustness 17 auxiliary real poles located at 0.17 have been added to the characteristic polynomial.

Figure 5 shows the characteristics of the output sensitivity function. The effect of auxiliary poles is illustrated. A zoom of the final characteristics is shown in Figure $6^{5}$.

\section{AdAPtive Control Design}

The adaptive approach uses the Youla-Kučera parametrization of the controller combined with the Internal Model Principle. The basic reference for this approach used in active vibration control is [6].

\footnotetext{
${ }^{5}$ The models and the robust controller can be downloaded from: http: //www.gipsa-lab.fr/ raul.melendez/
} 
A key aspect of this methodology is the use of the Internal Model Principle (IMP). It is supposed that $p(t)$ is a deterministic disturbance given by

$$
p(t)=\frac{N_{p}\left(q^{-1}\right)}{D_{p}\left(q^{-1}\right)} \cdot \delta(t),
$$

where $\delta(t)$ is a Dirac impulse and $N_{p}, D_{p}$ are coprime polynomials of degrees $n_{N_{p}}$ and $n_{D_{p}}$, respectively. In the case of stationary narrow-band disturbances, the roots of $D_{p}\left(z^{-1}\right)$ are on the unit circle.

Internal Model Principle [19]: The effect of the disturbance (15) upon the output

$$
y(t)=\frac{A\left(q^{-1}\right) S\left(q^{-1}\right)}{P\left(q^{-1}\right)} \cdot \frac{N_{p}\left(q^{-1}\right)}{D_{p}\left(q^{-1}\right)} \cdot \delta(t),
$$

where $D_{p}\left(z^{-1}\right)$ is a polynomial with roots on the unit circle and $P\left(z^{-1}\right)$ is an asymptotically stable polynomial, converges asymptotically towards zero iff the polynomial $S\left(z^{-1}\right)$ in the RS controller has the form (based on Eq. (9))

$$
S\left(z^{-1}\right)=D_{p}\left(z^{-1}\right) H_{S_{0}}\left(z^{-1}\right) S^{\prime}\left(z^{-1}\right) .
$$

Thus, the pre-specified part of $S\left(z^{-1}\right)$ should be chosen as $H_{S}\left(z^{-1}\right)=D_{p}\left(z^{-1}\right) H_{S_{0}}\left(z^{-1}\right)$ and the controller is computed solving

$$
P=A D_{p} H_{S_{0}} S^{\prime}+z^{-d} B H_{R_{0}} R^{\prime},
$$

where $P, D_{p}, A, B, H_{R_{0}}, H_{S_{0}}$ and $d$ are given ${ }^{6}$.

To build a direct adaptive controller, the Youla-Kučera parametrization of the controller is used. In the context of this paper, one considers a finite impulse response (FIR) filter of the form:

$$
Q\left(z^{-1}\right)=q_{0}+q_{1} z^{-1}+\cdots+q_{n_{Q}} z^{-n_{Q}},
$$

to which is associated the vector of parameters:

$$
\theta=\left[\begin{array}{llll}
q_{0} & q_{1} \ldots q_{n_{Q}}
\end{array}\right]^{T} .
$$

Under Youla-Kučera parametrization or $Q$-parametrization, the equivalent polynomials $R\left(z^{-1}\right)$ and $S\left(z^{-1}\right)$ of the controller $K\left(q^{-1}\right)$ take the form

$$
\begin{aligned}
& R\left(q^{-1}\right)=R_{0}+A \cdot Q \cdot H_{S_{0}} \cdot H_{R_{0}} \\
& S\left(q^{-1}\right)=S_{0}-q^{-d} B \cdot Q \cdot H_{S_{0}} \cdot H_{R_{0}},
\end{aligned}
$$

with

$$
\begin{aligned}
& R_{0}\left(z^{-1}\right)=r_{0}^{0}+r_{1}^{0} z^{-1}+\ldots+r_{n_{R}}^{0} z^{-n_{R_{0}}}=R_{0}^{\prime} \cdot H_{R_{0}} \\
& S_{0}\left(z^{-1}\right)=1+s_{1}^{0} z^{-1}+\ldots+s_{n_{S}}^{0} z^{-n_{S_{0}}}=S_{0}^{\prime} \cdot H_{S_{0}},
\end{aligned}
$$

where $A, B$ and $d$ correspond to the identified model of the secondary path, $R_{0}\left(z^{-1}\right), S_{0}\left(z^{-1}\right)$ are the central controller's polynomials, and $H_{S_{0}}, H_{R_{0}}$ are the controller's fixed parts ${ }^{7}$.

Using the output sensitivity function, the expression of the output can be written as:

\footnotetext{
${ }^{6}$ Of course, it is assumed that $D_{p}$ and $B$ do not have common factors.

${ }^{7}$ Under YK parametrization using a FIR structure for the Q filter, the closed loop poles defined by the central controller remain unchanged.
}

$$
y(t)=\frac{S_{0}}{P} \cdot w(t)-Q \cdot \frac{q^{-d} B H_{S_{0}} H_{R_{0}}}{P} \cdot w(t),
$$

with

$$
w(t)=A \cdot y(t)-q^{-d} B \cdot u(t)=A \cdot p(t)
$$

as a disturbance's observer. The objective is to find a value of $Q$ such that $y(t)$ is driven to zero.

7.

A block diagram of the adaptive scheme is given in Fig.

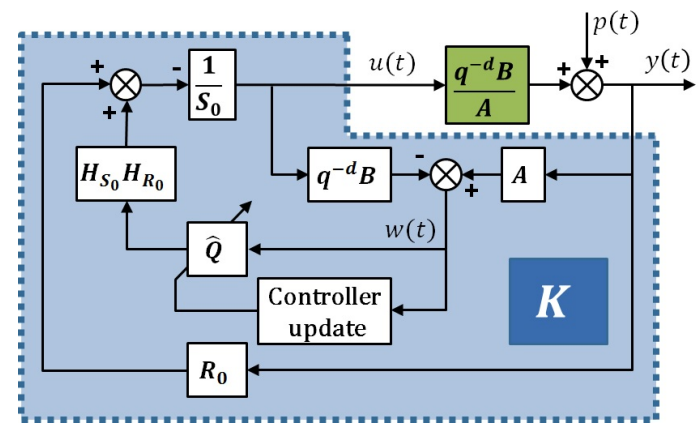

Fig. 7. Adaptive Youla-Kučera parametrization scheme.

The estimation of the polynomial $Q$ at time $t$ is denoted:

$$
\hat{Q}\left(t, q^{-1}\right)=\hat{q}_{0}(t)+\hat{q}_{1}(t) q^{-1}+\cdots+\hat{q}_{n_{Q}}(t) q^{-n_{Q}}
$$

and is characterized by the parameter vector ${ }^{8}$

$$
\hat{\theta}^{T}(t)=\left[\hat{q}_{0}(t) \hat{q}_{1}(t) \ldots \hat{q}_{n_{Q}}(t)\right]
$$

Since this is a regulation problem, $y(t)$ is expected to go towards zero and as such, it is an a priori adaptation error denoted $\varepsilon^{0}(t+1)$ for a given estimated polynomial $\hat{Q}\left(t, q^{-1}\right)$ :

$$
\varepsilon^{0}(t+1)=\frac{S_{0}}{P} \cdot w(t+1)-\hat{Q}(t) \frac{q^{-d} B^{*} H_{S_{0}} H_{R_{0}}}{P} \cdot w(t),
$$

with $B\left(q^{-1}\right)=q^{-1} \cdot B^{*}\left(q^{-1}\right)$. In a similar way, one can define an a posteriori error as:

$$
\varepsilon(t+1)=\frac{S_{0}}{P} \cdot w(t+1)-\hat{Q}(t+1) \frac{q^{-d} B^{*} H_{S_{0}} H_{R_{0}}}{P} \cdot w(t),
$$

which can be further expressed as

$$
\varepsilon(t+1)=[Q-\hat{Q}(t+1)] \cdot \frac{q^{-d} B^{*} H_{S_{0}} H_{R_{0}}}{P} \cdot w(t)+\eta(t+1)
$$

where $Q$ is the unknown optimal filter, and $\eta(t)$ tends asymptotically towards zero (see [20] for details).

Denoting filtered versions of the observer output $w(t)$ as

$$
\begin{gathered}
w_{1}(t)=\frac{S_{0}\left(q^{-1}\right)}{P\left(q^{-1}\right)} \cdot w(t) \\
w_{2}(t)=\frac{q^{-d} B^{*} H_{R_{0}} H_{S_{0}}}{P} \cdot w(t)
\end{gathered}
$$

${ }^{8}$ The order of the polynomial $\hat{Q}$ is related to the order of the denominator of the model of the disturbance $n_{D_{p}}$ as $n_{\hat{Q}}=n_{D_{p}}-1$. 
and

$$
\varphi^{T}(t)=\left[w_{2}(t) w_{2}(t-1) \ldots w_{2}\left(t-n_{Q}\right)\right],
$$

Eq. (31) can be rewritten as:

$$
\varepsilon(t+1)=\left[\theta^{T}-\hat{\theta}^{T}(t+1)\right] \cdot \varphi(t)+\eta(t+1) .
$$

where $\eta$ goes to zero. This type of equation allows immediately to develop an adaptation algorithm (see [20]):

$$
\begin{gathered}
\hat{\theta}(t+1)=\hat{\theta}(t)+F(t) \varphi(t) \varepsilon(t+1) \\
\varepsilon(t+1)=\frac{\varepsilon^{0}(t+1)}{1+\varphi^{T}(t) F(t) \varphi(t)} \\
\varepsilon^{0}(t+1)=w_{1}(t+1)-\hat{\theta}^{T}(t) \varphi(t) . \\
F(t+1)=\frac{1}{\lambda_{1}(t)}\left[\begin{array}{l}
F(t)-\frac{F(t) \varphi(t) \varphi^{T}(t) F(t)}{\lambda_{1}(t)} \\
\lambda_{2}(t) \\
\lambda^{T}(t) F(t) \varphi(t)
\end{array}\right] \\
0<\lambda_{1}(t) \leq 1 ; 0 \leq \lambda_{2}(t)<2 ; F(0)>0
\end{gathered}
$$

where $\lambda_{1}$ and $\lambda_{2}$ allow to obtain different profiles for the evolution of the adaptation gain $F(t)$. Finally the control to be applied is given by

$$
S_{0} \cdot u(t+1)=-R_{0} \cdot y(t+1)-H_{R_{0}} H_{S_{0}} \hat{Q}(t+1) \cdot w(t+1) .
$$

For the stability analysis of this algorithm, see [20].

In adaptive regulation applications, one uses in general the constant trace algorithm. In this case, $\lambda_{1}(t)$ and $\lambda_{2}(t)$ are automatically chosen at each step in order to ensure a constant trace of the gain matrix (constant sum of the diagonal terms):

$$
\operatorname{tr} F(t+1)=\operatorname{tr} F(t)=\operatorname{tr} F(0)=n G I
$$

in which $n$ is the number of parameters and $G I$ the initial adaptation gain. The matrix $F(0)$ has the form:

$$
F(0)=\left[\begin{array}{ccc}
G I & & 0 \\
& \ddots & \\
0 & & G I
\end{array}\right]
$$

The values of $\lambda_{1}(t)$ and $\lambda_{2}(t)$ at each sampling instant are determined from the equation:

$$
\operatorname{tr} F(t+1)=\frac{1}{\lambda_{1}(t)} \operatorname{tr}\left[F(t)-\frac{F(t) \phi(t) \phi^{T}(t) F(t)}{\alpha(t)+\phi^{T}(t) F(t) \phi(t)}\right]
$$

fixing the ratio $\alpha(t)=\lambda_{1}(t) / \lambda_{2}(t)$. This algorithm can be combined with the decreasing adaptation gain algorithm or with the variable forgetting factor algorithm for initialization [6]. One switches to the constant trace algorithm when the trace of the adaptation gain becomes equal or smaller than the assigned constant trace. Algorithms with constant scalar gain can be also implemented $(\mathrm{F}(\mathrm{t})=\mathrm{F}(0))$ but the results will be less good.

This scheme is implemented on top of the central controller which corresponds to the robust controller designed in Section V from which the BSF filters on $S_{y p}$ have been removed (preserving however the characteristics of $S_{u p}$ in high frequencies over $600 \mathrm{~Hz}$ for robustness reasons).

\section{EXPERIMENTAL RESULTS}

The robust controller and the adaptive controller have been tested on the experimental set-up described in Section II under several protocols.

\section{A. Interference test}

The protocol is as follows: for $1 \mathrm{~s}$, the system operates in open loop and without any disturbance in order to get a reference for the ambient noise. From $1 \mathrm{~s}$ to $10 \mathrm{~s}$, the test bench works in open loop, in the presence of two pairs of sinusoidal noise disturbances located at $170 \mathrm{~Hz}$ and $170.5 \mathrm{~Hz}$ and $285 \mathrm{~Hz}$ and $285.5 \mathrm{~Hz}$ respectively. At $10 \mathrm{~s}$, the loop is closed and the controller begins to counteract the disturbance effect. The frequencies of the four signals are then increased at $21 \mathrm{~s}$ by $10 \mathrm{~Hz}$. The corresponding new values are $180 \mathrm{~Hz}$ and $180.5 \mathrm{~Hz}$ for the first pair and $295 \mathrm{~Hz}$ and $295.5 \mathrm{~Hz}$ for the second pair (leaving the attenuation regions of the robust controller).

Fig. 8 shows the robust controller's performance for the interference experiment. As long as the disturbance frequencies are in the region of designed operation, a global attenuation of $39.86 \mathrm{~dB}$ is obtained (between $10 \mathrm{~s}$ and $21 \mathrm{~s}$ ). After $21 \mathrm{~s}$, since the frequencies of the disturbances are outside the region of designed operation, the performance is unsatisfactory achieving a global attenuation of only $7.94 \mathrm{~dB}$. 9 .

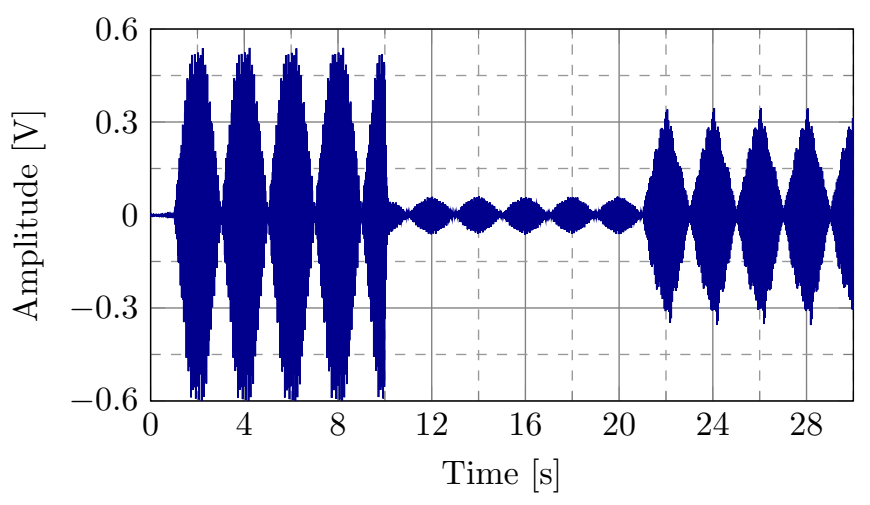

Fig. 8. Acoustic interference attenuation using a robust controller. Noise frequencies: $170+170.5 \mathrm{~Hz}, 285+285.5 \mathrm{~Hz}$ then $180+180.5 \mathrm{~Hz}$, $295+295.5 \mathrm{~Hz}$. Loop closed at $10 \mathrm{~s}$.

Fig. 9 presents the results for a similar test using the adaptive controller. The number of adjustable parameters in the Qfilter is $4\left(n_{Q}=3\right)$ and an adaptation algorithm with constant trace adaptation gain is used. The trace of the adaptation gain used was: $\operatorname{tr} F=0.03 \cdot\left(n_{Q}+1\right)$. It can be seen that after a negligible transient, a much better attenuation is obtained with respect to the robust controller between $10 \mathrm{~s}$ and $21 \mathrm{~s}$. The global attenuation obtained is $70.56 \mathrm{~dB}$. Excellent levels of attenuation are also obtained once the disturbances frequencies move away by $10 \mathrm{~Hz}$ (global attenuation $67.65 \mathrm{~dB}$ ), with a negligible adaptation transient ${ }^{10}$. Fig. 10 displays the

\footnotetext{
${ }^{9}$ Audio files available at http://www.gipsa-lab.fr/ raul.melendez/.

${ }^{10}$ Using $n_{Q}=7$ (8 adjustable parameters) does not improve the performance
} 
evolution of each Q-parameter with respect to time. From $0 \mathrm{~s}$ to $10 \mathrm{~s}$, all the parameters have values equal to zero since the controller is not working yet. Once the loop is closed, the Q-parameters take almost instantly stable mean values. At $21 \mathrm{~s}$, the change in frequencies leads to a quick adaptation towards the new values.

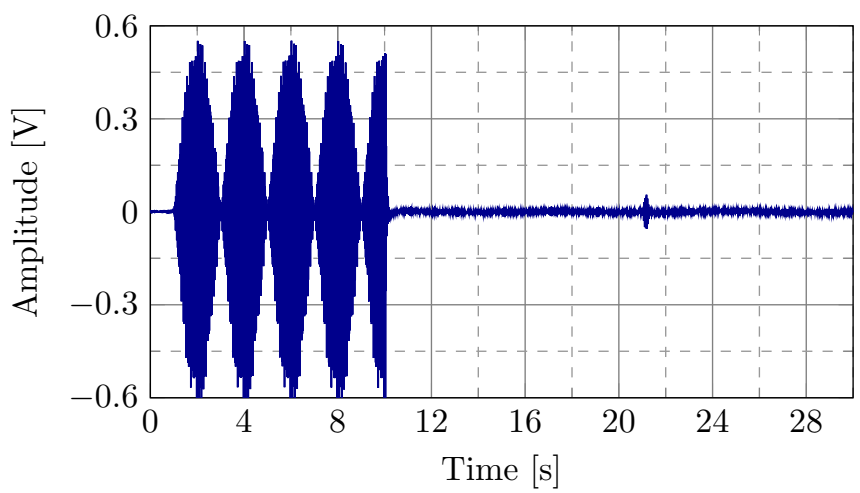

Fig. 9. Acoustic interference attenuation using an adaptive controller. Noise frequencies: $170+170.5 \mathrm{~Hz}, \quad 285+285.5 \mathrm{~Hz}$ then $180+180.5 \mathrm{~Hz}$, $295+295.5 \mathrm{~Hz}$. Loop closed at $10 \mathrm{~s}$.

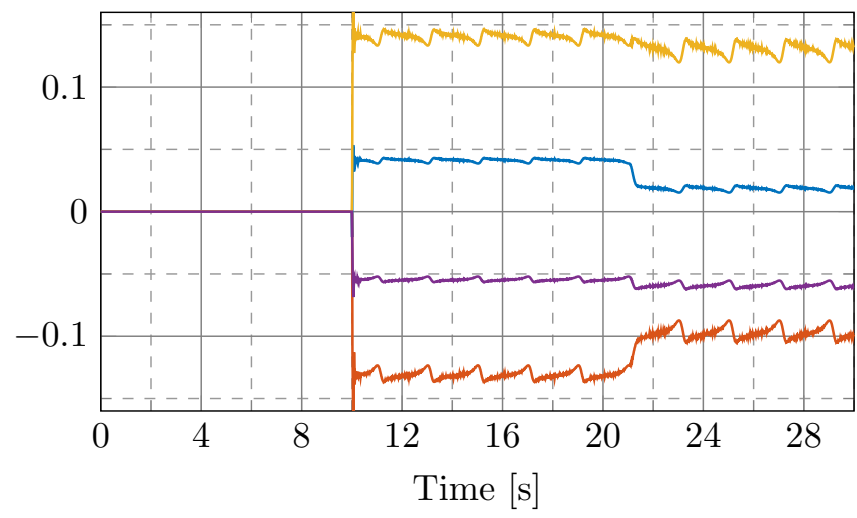

Fig. 10. Parameters evolution for acoustic interference test using an adaptive controller.

\section{B. Sinusoidal disturbances with continuously time- varying frequency}

In this experiment, a couple of tonal noise disturbances located at $160 \mathrm{~Hz}$ and $275 \mathrm{~Hz}$ are first applied to the system from $1 \mathrm{~s}$ to $6 \mathrm{~s}$. Then, their frequencies linearly increase till they reach the values of $180 \mathrm{~Hz}$ and $295 \mathrm{~Hz}$ correspondingly at $27 \mathrm{~s}$, after which their frequencies remain constant.

Fig. 11 displays a comparison between the system's residual noise when it is operated in open loop and in closed loop using the robust controller. As the frequencies move within the designed attenuation regions, a significant attenuation is obtained. However outside this zone, the performance is not satisfactory. Correspondingly, Fig. 12 displays the residual noise in open loop operation and with the adaptive controller. The levels of attenuation achieved are globally much better. The residual noise is comparable with the ambient noise

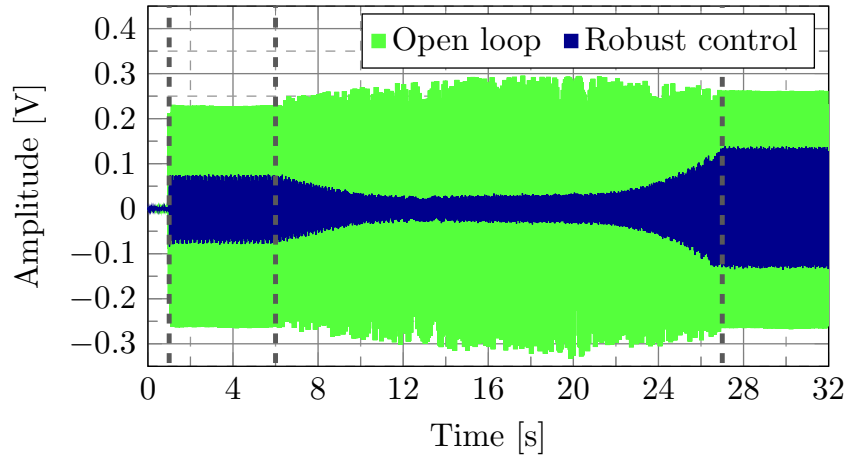

Fig. 11. Residual noise in open loop (green) and closed loop (blue) using a robust controller under the effect of tonal disturbances with variable frequencies.

measured between $0 \mathrm{~s}$ and $1 \mathrm{~s}$. The evolution of the parameters is shown in Fig. 13.

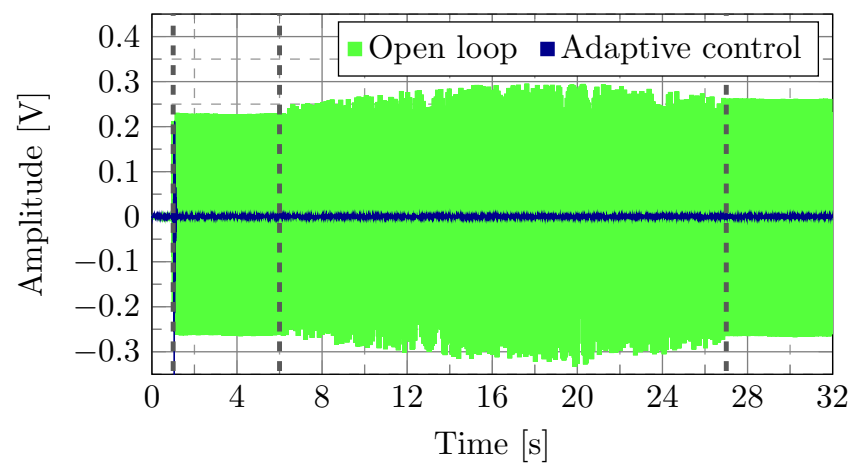

Fig. 12. Residual noise in open loop (green) and closed loop (blue) using an adaptive controller under the effect of tonal disturbances with variable frequencies.

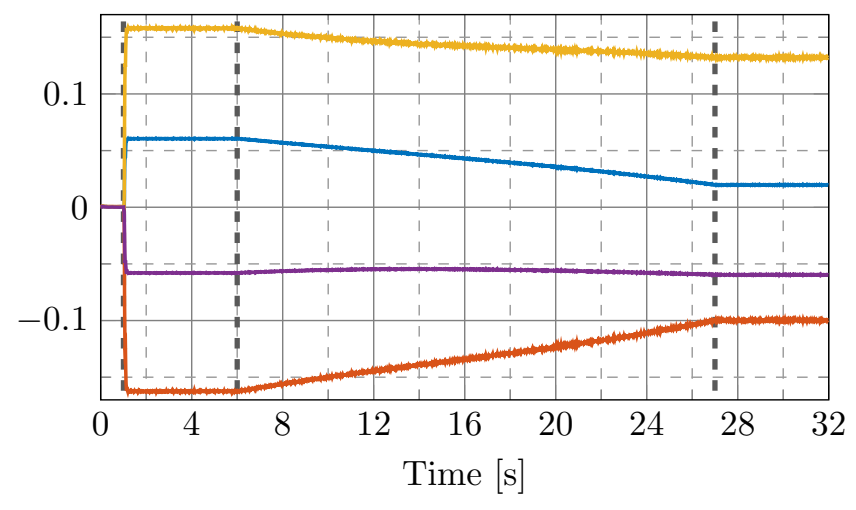

Fig. 13. Evolution of the controller parameters under the effect of tonal disturbances with variable frequency

\section{Step changes in frequencies}

In this experiment, step changes in the frequencies of a pair of tonal noise disturbances are considered, starting from their nominal values of $170 \mathrm{~Hz}$ and $285 \mathrm{~Hz}$. The steps are of $\pm 10 \mathrm{~Hz}$ and applied every $6.2 \mathrm{~s}$. The system is operated in open loop from 0 s to $1 \mathrm{~s}$. Fig. 14 displays the robust controller 
performance. When the disturbances' frequencies are inside the attenuation region of the controller, the attenuation is satisfactory. However for $-10 \mathrm{~Hz}$ and $+10 \mathrm{~Hz}$ steps, since one operates outside the designed regions of attenuation, the performance is unsatisfactory. The performance of the

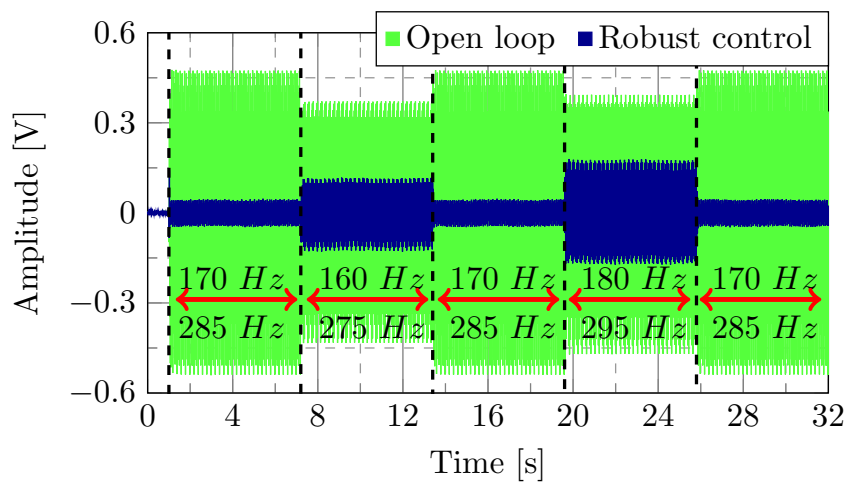

Fig. 14. Step changes in frequencies using the robust controller. Residual noise in open loop (green) and in closed loop (blue).

adaptive controller is illustrated in Fig. 15. The performance is almost the same for all frequencies values and the residual noise is close to the ambient noise. The adaptation transients are visible but very short. The same number of adjustable parameters and same adaptation gain as in the previous experiments have been used. The evolution of the Q-parameters is shown in Fig. 16

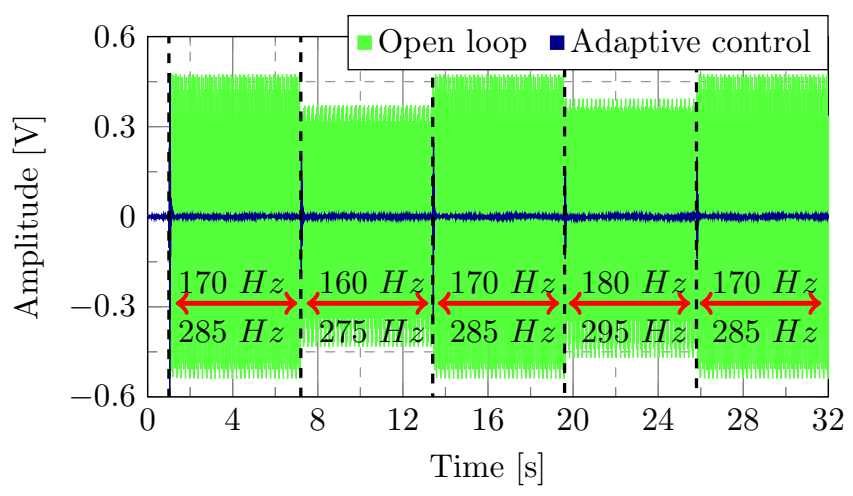

Fig. 15. Step changes in frequencies using the adaptive controller. Residual noise in open loop (green) and in closed loop (blue)..

\section{CONCLUSION}

The paper has shown that if the regions of frequency variations of multiple tonal noise disturbances are known and limited, an efficient robust feedback controller can be designed. Adding an adaptation feedback loop drastically enhances the performance of the robust controller in terms of achieved attenuation and expansion of the regions of attenuation in the frequency domain.

\section{REFERENCES}

[1] S. Elliott and P. Nelson, "Active noise control," Noise / News International, pp. 75-98, June 1994.

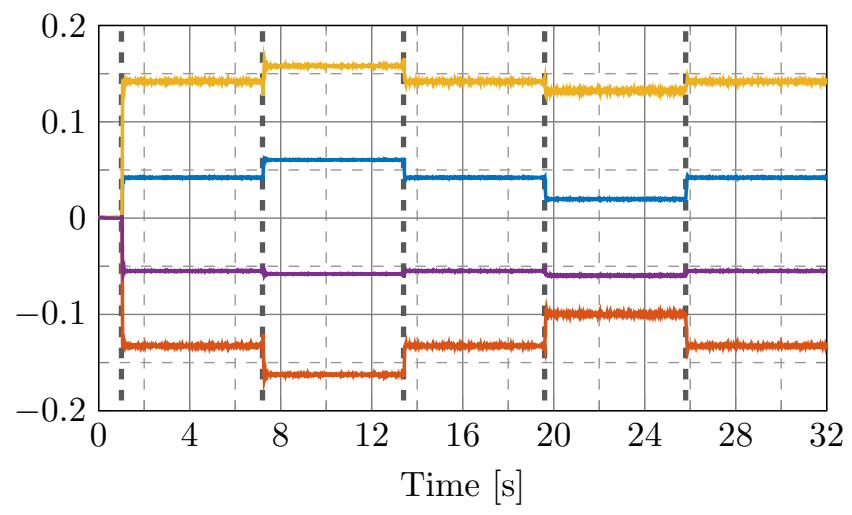

Fig. 16. Evolution of the parameters of the adaptive controller in the presence of step changes in disturbances frequencies.

[2] S. Elliott and T. Sutton, "Performance of feedforward and feedback systems for active control," IEEE Speech Audio Proces., vol. 4, no. 3, pp. 214-223, may 1996.

[3] S. Kuo and D. Morgan, "Active noise control: a tutorial review," Proceedings of the IEEE, vol. 87, no. 6, pp. 943 - 973, jun 1999.

[4] J. Zeng and R. de Callafon, "Recursive filter estimation for feedforward noise cancellation with acoustic coupling," Journal of Sound and Vibration, vol. 291, no. 3-5, pp. 1061 - 1079, 2006.

[5] B. Widrow and S. Stearns, Adaptive Signal Processing. Englewood Cliffs, New Jersey: Prentice-Hall, 1985.

[6] I. D. Landau, T.-B. Airimitoaie, A. Castellanos-Silva, and A. Constantinescu, Adaptive and Robust Active Vibration Control. London: Springer, 2016.

[7] J. C. Carmona and V. M. Alvarado, "Active noise control of a duct using robust control theory," IEEE-CST, vol. 8, no. 6, pp. 930-938, November 2000.

[8] A. S. S. Tansel Yucelen and F. Pourboghrat, "Active noise control in a duct using output feedback robust control techniques," in American Control Conference, 2010, pp. 3506-3511.

[9] C. A. S. L. Stefan Liebich, Daniel Rüschen, "Active noise cancellation in headphones by digital robust feedback control," in 24th European Signal Processing Conference (EUSIPCO), 2016, pp. 1843-1847.

[10] J. Shaw, "Adaptive control for sound and vibration attenuation: a comparative study," Journal of Sound and Vibration, vol. 235, no. 4 pp. 671-684, 2000.

[11] R. V. Harshad S. Sane and D. S. Bernstein, "Disturbance rejection using self-tuning armarkov adaptive control with simultaneous identification," IEEE-CST, vol. 9, no. 1, pp. 101-106, January 2001.

[12] S. Manikandan, "Literature survey of active noise control systems," Academic Open Internet Journal, vol. 17, 2006.

[13] F. Ben Amara, P. Kabamba, and A. Ulsoy, "Adaptive sinusoidal disturbance rejection in linear discrete-time systems - Part II: Experiments," Journal of Dynamic Systems Measurement and Control, vol. 121, pp. 655-659, 1999.

[14] J. Langer and I. D. Landau, "Combined pole placement/sensitivity function shaping method using convex optimization criteria," Automatica, vol. 35, no. 6, pp. 1111-1120, 1999.

[15] P. Nelson and S. Elliott, Active Control of Sound. Academic Press, 1993.

[16] B. J. Zimmer and S. P. Lipshitz, "An improved acoustic model for active noise control in a duct," Journal of Dynamic Systems, Measurement, and Control, vol. 125, pp. 382-395, September 2003.

[17] L. Ljung, System Identification - Theory for the User, 2nd ed. Englewood Cliffs: Prentice Hall, 1999.

[18] H. N. Duong and I. D. Landau, "An IV Based Criterion for Model Order Selection,” Automatica, vol. 32, no. 6, pp. 909-914, 1996.

[19] B. Francis and W. Wonham, "The internal model principle of control theory," Automatica, vol. 12, no. 5, pp. 457 - 465, 1976

[20] I. D. Landau, R. Lozano, M. M'Saad, and A. Karimi, Adaptive control, 2nd ed. London: Springer, 2011 